\title{
Molecular Transport between Two Phases in a Microchannel
}

\author{
Kiyoshi SATo, ${ }^{*}$ Manabu ToKeShI, ${ }^{* *}$ Tsuguo SAWAdA, ${ }^{*}$ and Takehiko KITAMORI ${ }^{* * * *}$ \\ *Department of Applied Chemistry, Graduate School of Engineering, The University of Tokyo, \\ Hongo, Bunkyo, Tokyo 113-8656, Japan \\ **Integrated Chemistry Project, Kanagawa Academy of Science and Technology, \\ Sakado, Takatsu, Kawasaki, Kanagawa 213-0012, Japan
}

(Received March 29, 2000; Accepted April 10, 2000)

Today, microfabricated devices (microchips) have attracted considerable attention because of their vast applicability and versatility. ${ }^{1,2}$ Most published reports utilizing microchips to separate and detect analysis of interest have concentrated on using electrokinetically driven separation schemes. ${ }^{3-7}$ Investigations from a different standpoint have been few in number. ${ }^{89}$ However, microchips offer advantages concerning the scale merits of microspace, such as a short diffusion distance and the high interface-to-volume ratio, the specific interface area; we thus considered that they are an ideal tool to study molecular transport between two different phases, i.e., solvent extraction.

In the present paper, we report on the first demonstration using a microchip to study molecular transport between two phases.

\section{Experimental}

We have described the experimental apparatus and the principle of measurement for the thermal lens microscope (TLM) in detail previously. ${ }^{10-12}$ The excitation beam was an $\mathrm{Ar}^{+}$laser of $488.0 \mathrm{~nm}$ which was mechanically chopped by a light chopper at $1.69 \mathrm{kHz}$. The probe beam was a He-Ne laser of $632.8 \mathrm{~nm}$. Two laser beams were introduced coaxially into an optical microscope and tightly focused into a sample in a microchannel (100 $\mu \mathrm{m} \times 150 \mu \mathrm{m}$ cross section) by an $20 \times 0.65$ NA objective lens. The excitation beam passing through the sample was separated from the probe beam by an optical narrow band-pass filter and a diffraction grating. The intensity of the transmitted probe beam after passing through a pinhole was detected by a photodiode. The photodiode current, amplified by a low-noise preamplifier $(\times 100)$, was fed into a lock-in amplifier.

The fabrication method for our glass chip was also previously published. ${ }^{11,12}$ Figure 1 shows the layout and dimensions of the glass chip.

Nickel(II) chloride, dimethylglyoxime, ethanol, sodium acetate, acetic acid, hydrochloric acid, and chloroform were obtained from Wako Pure Chemical Industries, Ltd. and were used as received. Chloroform was of analytical grade. The other

$\doteqdot$ To whom correspondence should be addressed.

K.S. present address: Clean Technology R\&D Center, Minebea Co., Ltd., Kitasaku, Nagano 389-0293, Japan. chemicals were of guaranteed grade, and the water used was from a Millipore Milli-Q system. A $2.5 \mathrm{mM}$ nickel(II) stock solution was prepared by dissolving nickel(II) chloride in a small amount of hydrochloric acid and diluting with water. Aqueous solutions of $\mathrm{Ni}(\mathrm{II})$ were prepared at concentrations of $2.5-50$ $\mu \mathrm{M}$ by successive dilution with water. A stock solution of dimethylglyoxime was prepared by dissolving dimethylglyoxime in a small amount of ethanol and diluting to $1 \mathrm{mM}$ with water. An aqueous buffer solution was prepared as follows. Acetic acid $(2.4 \mathrm{ml})$ and $12.25 \mathrm{mg}$ of sodium acetate were mixed and diluted to $250 \mathrm{ml}$ with water. The Ni-complex sample solutions were prepared with a 1:1:1 mixing volume ratio of the nickel(II) solution, buffer solution and dimethylglyoxime solution.

\section{Results and Discussion}

A Ni-dimethylglyoxime complex solution and chloroform in each reservoir were introduced into the solvent-extraction region by suction, as shown in Fig. 1. The two solutions did not mix with each other, and a liquid-liquid (aqueous/organic) interface produced at the center of the microchannel was present for several minutes. In the case of ordinary solvent extraction using a separatory funnel, the two solutions in the separatory

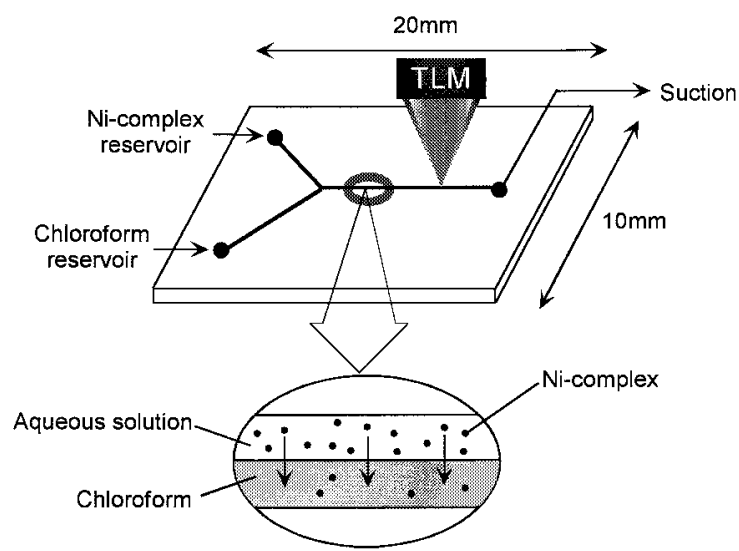

Fig. 1 Schematic illustration of the integrated micro-extraction system. 


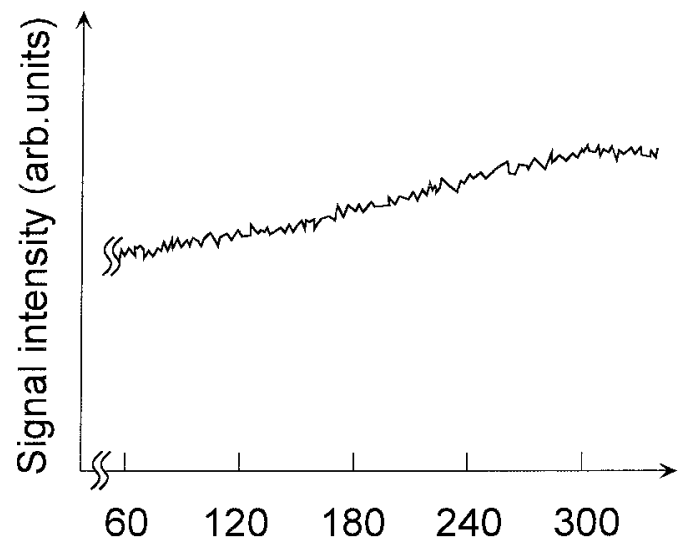

Time (s)

Fig. 2 Temporal behavior of the TLM signal showing extraction of the Ni(II)-complex. The concentration of $\mathrm{Ni}(\mathrm{II})$ solution was $10 \mu \mathrm{M}$.

funnel are separated horizontally by the difference in their specific gravities. In the microchannel, the liquid-liquid interface was formed parallel to its sidewalls, because the influences of surface tension and friction force are much stronger than the specific-gravity difference in the microspace.

The Ni-complex was gradually extracted from the aqueous solution into the chloroform, where it was detected using TLM. The Ni-complex was detected at the center of chloroform phase, just halfway between interface and sidewall, $4 \mathrm{~mm}$ downstream from the intersection point in the Y-shaped microchannel. The TLM signal increased with time and then remained constant after about $5 \mathrm{~min}$. A typical time profile of the TLM signal is shown in Fig. 2. In a blank test of chloroform and aqueous solution (without nickel(II) chloride), the TLM signal did not change with time; it remained at the background signal level. Thus, the increment of the TLM signal with time in Fig. 2 corresponds to the quantity of nickel complex which was transported from the water phase. This is the first demonstration of molecular transport between two different phases in a microchannel. The transportation time of Ni-complex from the aqueous solution into chloroform is estimated to be several tens of seconds based on the diffusion coefficient ${ }^{13}$ and the microchannel width. The difference between the observed and estimated values may result from the influence of interfacial adsorption of the complex and/or interfacial potential.

The dependence of the TLM signal on the concentration of $\mathrm{Ni}(\mathrm{II})$ is shown in Fig. 3. For a comparison, Ni-complex was extracted from aqueous solution into chloroform with a separatory funnel (aqueous solution: $20 \mathrm{ml}$, chloroform: $20 \mathrm{ml}$ ), and introduced and then detected in the same microchannel. This result is also shown in Fig. 3. Both show good linear calibration curves. However, the TLM signal intensity in the microchannel is about 3-times larger than that in the separatory funnel. The reason for this is not clear. The introduction method of the samples is now under investigation as being the most probable cause.

Although further investigations are necessary, the present study indicates that molecular transport between two different phases, i.e., solvent extraction, in the microchannel is possible.

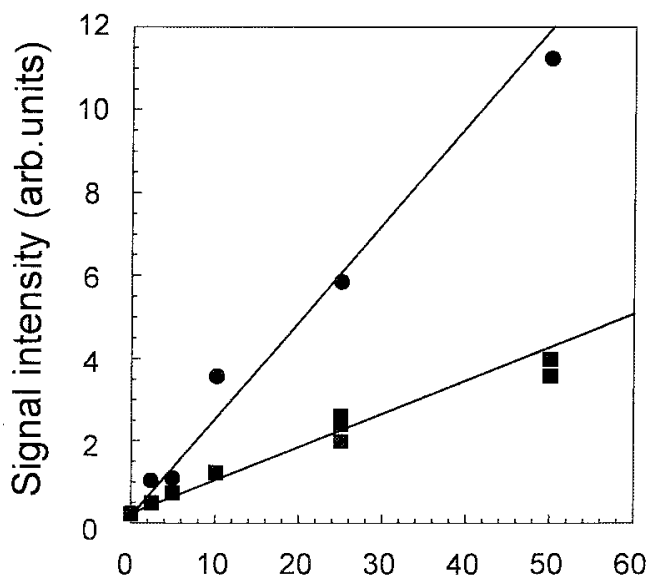

\section{Concentration of $\mathrm{Ni}(\mathrm{II})(\mu \mathrm{M})$}

Fig. 3 Dependence of the TLM signal on the concentration of $\mathrm{Ni}(\mathrm{II})$ : microchannel (•), separatory funnel ( $\boldsymbol{\bullet})$

The microchannel structure and TLM detection provide a good laboratory tool for making small experimental devices which could be used in a wide range of research fields, including chemical and biochemical analyses.

This work was financially supported by the Grant-in-Aid for Specially Promoted Research (No.07102004) and for University and Society Collaboration (No.11794006) from the Ministry of Education, Science, Sports and Culture of Japan.

\section{References}

1. D. J. Harrison and A. von den Berg, "Micro Total Analysis Systems '98", 1998, Kluwer Academic Publishers, Dordrecht, Boston, London.

2. M. Freemantle, $C \& E$ News, February 22, 1999, 27.

3. A. Manz, D. J. Harrison, E. M. J. Verpoorte, J. C. Fettinger, A. Paulus, H. Lüdi, and H. M. Widmer, J. Chromatogr., 1992, 593, 253.

4. S. C. Jacobson, R. Hergenröder, L. B. Koutny, and J. M. Ramsey, Anal. Chem., 1994, 66, 2369.

5. D. E. Raymond, A. Manz, and H. M. Widmer, Anal. Chem., 1994, 66, 2858.

6. C. S. Effenhauser, A. Paulus, A. Manz, and H. M. Widmer, Anal. Chem., 1994, 66, 2949.

7. A. W. Moore Jr., S. C. Jacobson, and J. M. Ramsey, Anal. Chem., 1995, 67, 4184.

8. H. A. Weigl and P. Yager, Science, 1999, 283, 346.

9. P. J. Kenis, R. F. Ismagilov, and G. M. Whitesides, Science, 1999, 285, 83.

10. K. Sato, H. Kawanishi, M. Tokeshi, T. Kitamori, and T. Sawada, Anal. Sci., 1999, 15, 525.

11. K. Sato, M. Tokeshi, T. Kitamori, and T. Sawada, Anal. Sci., 1999, 15, 641.

12. M. Tokeshi, M. Uchida, K. Uchiyama, T. Sawada, and T. Kitamori, J. Luminescence, 1999, 83-84, 261.

13. D. R. Lide, "CRC Handbook of Chemistry and Physics 78th Edition”, 1997, CRC Press, Boca Raton, New York, 5 - 93. 